\title{
Infinite Iterated Function Systems Depending on a Parameter
}

by

\author{
Ludwik JAKSZTAS
}

Presented by Stanisław KWAPIEŃ

\begin{abstract}
Summary. This paper is motivated by the problem of dependence of the Hausdorff dimension of the Julia-Lavaurs sets $J_{0, \sigma}$ for the map $f_{0}(z)=z^{2}+1 / 4$ on the parameter $\sigma$. Using homographies, we imitate the construction of the iterated function system (IFS) whose limit set is a subset of $J_{0, \sigma}$, given by Urbański and Zinsmeister. The closure of the limit set of our IFS $\left\{\phi_{\sigma, \alpha}^{n, k}\right\}$ is the closure of some family of circles, and if the parameter $\sigma$ varies, then the behavior of the limit set is similar to the behavior of $J_{0, \sigma}$. The parameter $\alpha$ determines the diameter of the largest circle, and therefore the diameters of other circles.
\end{abstract}

We prove that for all parameters $\alpha$ except possibly for a set without accumulation points, for all appropriate $t>1$ the sum of the $t$ th powers of the diameters of the images of the largest circle under the maps of the IFS depends on the parameter $\sigma$. This is the first step to verifying the conjectured dependence of the pressure and Hausdorff dimension on $\sigma$ for our model and for $J_{0, \sigma}$.

1. Introduction. This paper is devoted to construction of some infinite conformal iterated function system (IFS, see [4] and Section 3) which consists of restrictions, to a domain $X$, of homographies depending on two parameters $(\sigma$ and $\alpha)$. The motivation for writing this paper has been an open problem of dependence of the Hausdorff dimension of the Julia-Lavaurs sets on the parameter for $f_{0}(z)=z^{2}+1 / 4$ (see Section 2, with no technical connection with the rest of the paper).

2000 Mathematics Subject Classification: Primary 37F45; Secondary 37D35.

Key words and phrases: Hausdorff dimension, iterated function system, Julia-Lavaurs sets.

Partially supported by Polish KBN Grant No. 2PO3A03425. 
The domain $X$ of our IFS is a subset of the closed disk $\bar{B}(1 / 2,1 / 2)$. We will use the homographies

$$
\begin{aligned}
& f(z)=\frac{z}{i z+1}=\frac{1}{i+1 / z}, \\
& s(z)=1-z,
\end{aligned}
$$

which are automorphisms of $\bar{B}(1 / 2,1 / 2)$ and do not depend on parameters, and

$$
g_{\sigma, \alpha}(z)=\frac{z}{\left(\frac{1-\alpha}{\alpha}+i \sigma\right) z+1}, \quad \sigma \in \mathbb{R}, \alpha \in(0,1) .
$$

We have $g_{\sigma, \alpha}(\bar{B}(1 / 2,1 / 2)) \subset \bar{B}(1 / 2,1 / 2)$, but the IFS will be defined only for $\alpha \in(0,1 / 2]$ and in this case $g_{\sigma, \alpha}(\bar{B}(1 / 2,1 / 2)) \subset \bar{B}(1 / 4,1 / 4)$.

Notice that in the coordinates $1 / z$ the map $f$ is translation by $i$, while $g_{\sigma, \alpha}$ is translation by $(1-\alpha) / \alpha+i \sigma$.

The family of maps $\phi_{\sigma, \alpha}^{n, k}: X \rightarrow X$, which will form the IFS, is defined as follows:

$$
\phi_{\sigma, \alpha}^{n, k}=g_{\sigma, \alpha}^{n} \circ f^{k} \circ s
$$

if $n \geq 1, k \in \mathbb{Z}$ or $n=0, k \leq-3$, and

$$
\phi_{\sigma, \alpha}^{n, k}=g_{\sigma, \alpha}^{n} \circ f^{k} \circ s \circ f
$$

if $n=0, k \geq 2$. The set of pairs $(n, k)$ for which $\phi_{\sigma, \alpha}^{n, k}$ is defined is denoted by $I$.

The closure of the limit set of the IFS (we add only countably many points) is the closure of a certain family of circles. The parameter $\alpha$ is the diameter of the largest circle contained in the closure of the limit set, namely $S(\alpha / 2, \alpha / 2)$, and therefore controls the diameters of other circles. The parameter $\sigma$ influences the position of some families of circles (analogy with the Julia-Lavaurs sets).

Choosing an arbitrary point $x \in X$ and $t>0$ we can consider the pressure $P(t)$ with the use of the derivative of $\phi_{\sigma, \alpha}^{n, k}$ (see (3.2)). Then the relevant Hausdorff dimension is $t_{0}$ such that $P\left(t_{0}\right)=0$.

If the homograpies $\phi_{\sigma, \alpha}^{n, k}$ are replaced by conformal affine functions mapping $S(\alpha / 2, \alpha / 2)$ onto the image of $S(\alpha / 2, \alpha / 2)$ under $\phi_{\sigma, \alpha}^{n, k}$, then the derivatives of the affine functions are quotients of the diameters of suitable circles. If the IFS obtained in this way satisfies conditions from Section 3, then the Hausdorff dimension of its limit set depends on the value of the sum of the $t$ th powers of these quotients, as a function of $t$ (the dimension $t_{0}$ is then a zero of the logarithm of this sum). We will prove that this sum really depends on $\sigma$, for all parameters $\alpha$ except possibly for $\alpha$ 's from a set without accumulation points, which gives us in this ideal situation the dependence of the Hausdorff dimension on $\sigma$. 


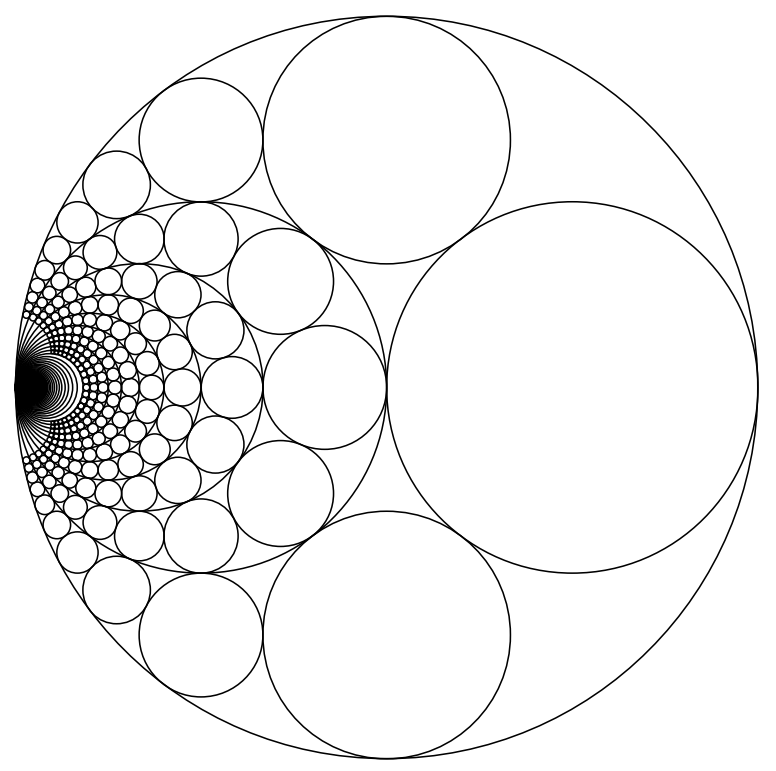

Fig. 1. Circles

We have not been able to verify the OSC condition. Without OSC, $t_{0}$ is called the similarity dimension, so we prove that the similarity dimension in the affine case is not constant.

This is a part of the author's $\mathrm{PhD}$ thesis written under the supervision of Professor Feliks Przytycki.

2. Analogy with the Julia-Lavaurs sets. For a polynomial $f$ we define the filled-in Julia set $K(f)$ as the set of points that do not escape to infinity under iteration of $f$. The boundary of $K(f)$ is called the Julia set of $f$. Let us consider the set of polynomials of the form $f_{\varepsilon}(z)=z^{2}+1 / 4+\varepsilon$. We obtain the function $\varepsilon \mapsto J\left(f_{\varepsilon}\right)$ with values in the set $\mathcal{K}(\mathbb{C})$ of compact subsets of $\mathbb{C}$, equipped with the Hausdorff metric. We will also consider the function $\varepsilon \mapsto d(1 / 4+\varepsilon)$, where $d(1 / 4+\varepsilon)$ denotes the Hausdorff dimension of $J\left(f_{\varepsilon}\right)$. It follows from [1] and [5] that the functions $\varepsilon \mapsto J\left(f_{\varepsilon}\right)$ and $\varepsilon \mapsto d(1 / 4+\varepsilon)$ are continuous and real-analytic respectively on the set of parameters $\varepsilon$ for which $f_{\varepsilon}$ is hyperbolic.

Now let $\varepsilon \in \mathbb{R}^{+} \cup\{0\}$. If $\varepsilon \in \mathbb{R}^{+}$then $f_{\varepsilon}$ is hyperbolic, and $J\left(f_{\varepsilon}\right)$ is homeomorphic to the Cantor set. The function $f_{0}$ is not hyperbolic since $f_{0}(1 / 2)=1 / 2$, and $f_{0}^{\prime}(1 / 2)=1$. It is interesting to study the behaviour of $J\left(f_{\varepsilon}\right)$ when $\varepsilon \searrow 0$ (see [1]). We know that the function $\varepsilon \mapsto J\left(f_{\varepsilon}\right)$ is not rightcontinuous at zero. The possible limits of $J\left(f_{\varepsilon}\right)$ in $\mathcal{K}(\mathbb{C})$ which occur after passing to a subsequence are called Julia-Lavaurs sets. These sets depend 
on the parameter $\sigma \in \mathbb{R}$ and are denoted by $J_{0, \sigma}$, with $J_{0, \sigma}=J_{0, \sigma^{\prime}}$ if and only if $\sigma-\sigma^{\prime} \in \mathbb{Z}$.

The Julia-Lavaurs sets can also be defined as the closures of $J\left(f_{0}\right)$ and the union of all preimages of $J\left(f_{0}\right)$ under $g_{\sigma}^{-n}$, for some special map $g_{\sigma}$ : Int $K\left(f_{0}\right) \rightarrow \mathbb{C}$ (Lavaurs map, see [1]). For all $\sigma \in \mathbb{R}$ we have $J\left(f_{0}\right) \subset J_{0, \sigma} \subset$ $K\left(f_{0}\right)$ (because $\left.g_{\sigma}^{-1}\left(\operatorname{Int} K\left(f_{0}\right)\right) \subset \operatorname{Int} K\left(f_{0}\right)\right)$. The map $g_{\sigma}$ has infinite degree on Int $K\left(f_{0}\right)$, and $\sigma(\bmod 1)$ is relevant to the Lavaurs map between Ecalle cylinders. Furthermore, using $f_{0}, g_{\sigma}$ and symmetry with respect to zero (the sets $J_{0, \sigma}$, just like $J_{0}$, are symmetrical with respect to zero), we can obtain a subset of $J_{0, \sigma}$ as the closure of the limit set of some infinite conformal IFS (see [6] and [2]). The images of this subset under the action of the iterates of $f_{0}$ cover the set $J_{0, \sigma}$ (beginning with the second iteration).

The function $\varepsilon \mapsto d(1 / 4+\varepsilon)$ is not continuous at zero either (see [2]). If we choose a sequence of parameters $\varepsilon_{n}$ so as to obtain convergence of $J\left(f_{\varepsilon_{n}}\right)$ in the Hausdorff metric to $J_{0, \sigma}$ for some $\sigma$ (precisely $\frac{-\pi}{\sqrt{\varepsilon_{n}}}(\bmod 1)$ $\rightarrow \sigma)$, then we also obtain convergence of the Hausdorff dimension of $J\left(f_{\varepsilon_{n}}\right)$ to the Hausdorff dimension of $J_{0, \sigma}$. It is a famous problem whether the Hausdorff dimension of $J_{0, \sigma}$ depends on the parameter $\sigma$, and whether the limit $\lim _{\varepsilon \backslash 0} d(1 / 4+\varepsilon)$ exists.

This problem has been a motivation for this paper; we will study simpler sets, though obtained in a similar way. Because we are interested in the Hausdorff dimension, we will imitate the construction of the IFS generating a subset of $J_{0, \sigma}$, and the functions used there will be replaced by homographies (1.1)-(1.3). The closure of the limit set (which will be denoted by $C_{\sigma, \alpha}$ ) is a subset of $\bar{B}(1 / 2,1 / 2)$. Then the symmetry with respect to 0 is replaced by the symmetry $s$ with respect to $1 / 2, f_{0}$ is replaced by $f$, and instead of $g_{\sigma}^{-1}$ we take $g_{\sigma, \alpha}$. Notice that similarly to the case of $f_{0}$ and $g_{\sigma}$ in [1], we have

$$
g_{\sigma, \alpha} \circ f=f \circ g_{\sigma, \alpha}=g_{\sigma+1, \alpha} .
$$

The parameter $\sigma$ plays a similar role to that in the case of Julia-Lavaurs sets, while $\alpha$ is an additonal parameter which controls the diameters of circles.

In the case of Julia-Lavaurs sets the closure of the limit set is a subset of $J_{0, \sigma}$, and the whole set may be obtained by taking its images under iterates of $f_{0}$. In our case, if we take the union of the images of $C_{\sigma, \alpha}$ under $f^{k}$ (it is enough to take $k=0, \ldots, 5$ ), we may obtain the sets $\widetilde{C}_{\sigma, \alpha}$. The set $J_{0, \sigma}$ is the closure of the union of the preimages of $J\left(f_{0}\right)$ under $g_{\sigma}^{-n}$. The sets $\widetilde{C}_{\sigma, \alpha}$ can be defined in a similar way: $\widetilde{C}_{\sigma, \alpha}$ is the closure of the union of the images of $S(1 / 2,1 / 2)$ under all compositions of the maps $f^{k}, g_{\sigma, \alpha}^{n}$ and $s$, where $k \in \mathbb{Z}, n \in \mathbb{N}$ (in the case of Julia-Lavaurs sets, considering $f_{0}$ and symmetry with respect to zero does not add anything new). 
3. Conformal IFS. In this section, following [4], we define an IFS and state some important conditions.

Let $I$ be a countable set with at least two elements, and let $X \subset \mathbb{R}^{d}$ for some $d \geq 1$. Let $S=\left\{\phi_{i}: X \rightarrow X\right\}_{i \in I}$ be a set of injective contractions for which there exists $0<s<1$ such that

$$
\left|\phi_{i}(x)-\phi_{i}(y)\right| \leq s|x-y|
$$

for all $x, y \in X$ and $i \in I$. Any such family $S$ is called an Iterated Function System (IFS).

If $\omega \in I^{n}$ then $\omega=\left(\omega_{1}\right.$, ldots, $\left.\omega_{n}\right)$ and $\phi_{\omega}$ will be defined as follows:

$$
\phi_{\omega}=\phi_{\omega_{1}} \circ \cdots \circ \phi_{\omega_{n}} .
$$

The limit set of the system $S$ is the only set $J_{S}$ which satisfies

$$
J_{S}=\bigcup_{i \in I} \phi_{i}\left(J_{S}\right) \text {. }
$$

We say that the system $S$ satisfies the Open Set Condition (OSC) if there exists a non-empty open set $U \subset X$ (in the topology of $X$ ) such that $\phi_{i}(U) \subset U$ for all $i \in I$ and $\phi_{i}(U) \cap \phi_{j}(U)=\emptyset$ for $i, j$ distinct.

Later on we will limit our considerations to the case $d=2\left(X \subset \mathbb{R}^{2}\right)$.

An IFS consisting of conformal maps will be called conformal. The following conditions are also important (see [4]):

(1) $X$ is a compact, connected subset of $\mathbb{R}^{2}$, and OSC is satisfied for $U=\operatorname{Int}_{\mathbb{R}^{d}}(X)$.

(2) There exist $\alpha, l>0$ such that for every $x \in \partial X$ there exists an open cone included in $\operatorname{Int}(X)$ with vertex $x$, altitude $l$, and central angle $\alpha$.

(3) There exists an open connected set $V$ with $X \subset V \subset \mathbb{R}^{2}$ such that each $\phi_{i}$ conformally extends onto $V$. Then $D \phi_{j}(x)$ and also $D \phi_{\omega}(x)$ are similarities; their scaling coefficients are denoted by $\left|\phi_{j}^{\prime}(x)\right|$ and $\left|\phi_{\omega}^{\prime}(x)\right|$.

(4) Bounded Distortion Property (BDP): There exists a constant $K \geq 1$ such that $\left|\phi_{\omega}^{\prime}(x)\right| \leq K\left|\phi_{\omega}^{\prime}(y)\right|$ for all $n \in \mathbb{N}, \omega \in I^{n}$ and $x, y \in V$.

For $t>0$ we may define a number $P(t)$ called the topological pressure of the parameter $t$ by

$$
P(t)=\lim _{n \rightarrow \infty} \frac{1}{n} \log \sum_{\omega \in I^{n}}\left|\phi_{\omega}^{\prime}(x)\right|^{t},
$$

and the limit does not depend on $x \in X$. On the set where it is finite, the function $P(t)$ is continuous, strictly decreasing to $-\infty$ and convex.

Let $h_{S}$ denotes the Hausdorff dimension of $J_{S}$. 
ThEOREM 3.1 ([4]). For a system $S$ which satisfies the above conditions, we have

$$
h_{S}=\inf \{t \geq 0: P(t)<0\} .
$$

COROLlary $3.2([4])$. Under the above assumptions, if the system $S$ consists of affine conformal maps, then $P(t)=\log \sum_{i \in I}\left|\phi_{\omega}^{\prime}(x)\right|^{t}$, and

$$
h_{S}=\inf \left\{t \geq 0: \sum_{i \in I}\left|\phi_{i}^{\prime}(x)\right|<1\right\} .
$$

If there exists $t$ for which $0<P(t)<\infty$ then the system is called strongly regular.

4. Construction. In this section we construct a set $X \subset \bar{B}(1 / 2,1 / 2)$, and maps $\phi_{\sigma, \alpha}^{n, k}: X \rightarrow X$. In the next section we will prove that these maps form an IFS.

The map $\mathcal{I}(z)=1 / z$ (called inversion) maps $\bar{B}(1 / 2,1 / 2)$ onto the halfplane $\{z: \operatorname{Re}(z) \geq 1\}$ together with the point at infinity. In many cases it will be useful to consider the half-plane rather than the disk.

In order to define $X$ we first describe a set $Y \subset\{z: \operatorname{Re}(z) \geq 1\}$ (the same for all $\alpha \in(0,1 / 2]$ and $\sigma \in \mathbb{R})$, and take $X=\mathcal{I}(Y)$. We will also consider maps conjugated to $f, g_{\sigma, \alpha}, s$ by $\mathcal{I}$, which help us to prove some properties of the IFS.

The map $f$ is conjugate to translation by $i$, while $g_{\sigma, \alpha}$ is conjugate to translation by $(1-\alpha) / \alpha+i \sigma$. Set

$$
F_{\sigma, \alpha}^{n, k}:=g_{\sigma, \alpha}^{n} \circ f^{k},
$$

where $n \in \mathbb{N}$ (we assume that $0 \in \mathbb{N}$ ), $k \in \mathbb{Z}$. Then $F_{\sigma, \alpha}^{n, k}$ is conjugate to

$$
T_{\sigma, \alpha}^{n, k}(z):=z+\frac{1-\alpha}{\alpha} n+i k+i \sigma n=\left(\mathcal{I} \circ F_{\sigma, \alpha}^{n, k} \circ \mathcal{I}\right)(z) .
$$

Next, the map $s$ is conjugated by $\mathcal{I}$ to

$$
\widetilde{\mathcal{I}}(z):=\mathcal{I} \circ s \circ \mathcal{I}(z)=1+\frac{1}{z-1}=1+\mathcal{I}(z-1) .
$$

Hence $\widetilde{\mathcal{I}}$ is $\mathcal{I}$ conjugated by translation by one.

Now we give a construction which will be used for the definition of $Y$. The half-plane $\{z: \operatorname{Re}(z) \geq 1\}$ with the point at infinity will be denoted by $\bar{H}$.

Let closed disks $\bar{B}_{1}, \bar{B}_{2}, \bar{B}_{3} \subset \bar{H}$ have pairwise empty interiors, be pairwise tangent, and tangent to $\partial \bar{H}$ (as disks we take sets $\{z: \operatorname{Re}(z) \geq C\}$ $\cup\{\infty\})$. For any such $\bar{B}_{1}, \bar{B}_{2}$ and $\bar{B}_{3}$ we construct a uniquely determined sequence of disks $\bar{B}_{n}, n \geq 1$. If $n>3$ and we have chosen $\bar{B}_{1}, \ldots, \bar{B}_{n-1}$, let $\bar{B}_{n}$ be the disk which is tangent to $\bar{B}_{n-2}, \bar{B}_{n-1}, \partial \bar{H}$, and is disjoint from 


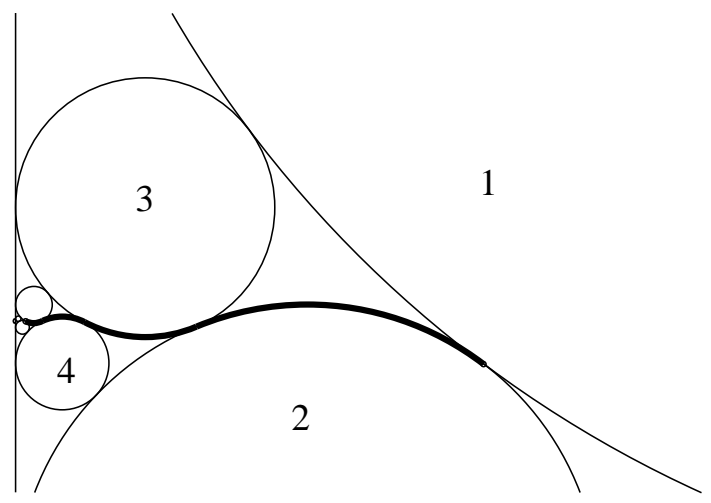

Fig. 2. Tangent disks and the curve $\gamma$

the interiors of all disks previously chosen. In this way we get a sequence of disks tending to a point $b \in \partial \bar{H}$.

We also define a curve $\gamma_{B}$ which separates the disks with even indices from those with odd indices. First we take the arc of the boundary $\bar{B}_{2}$ between the points of tangency with $\bar{B}_{1}$ and $\bar{B}_{3}$ (we take the component without the point of tangency with $\bar{B}_{4}$ ). Next, for $n \geq 2$ we take the part of the boundary of $\bar{B}_{n}$ between the points of tangency with $\bar{B}_{n-1}$ and $\bar{B}_{n+1}$ (containing the point of tangency with $\bar{B}_{n+2}$ ). After taking the closure, we obtain a curve joining the point of tangency of $\bar{B}_{1}$ and $\bar{B}_{2}$ to a point $b \in \partial \bar{H}$.

Now we are able to define the set $Y \subset \bar{H}$.

The set $Y$ is closed, $Y \cap \mathbb{C}$ is unbounded, in particular contains the half-plane $\{z: \operatorname{Re}(z) \geq 2\}$. The boundary of $Y$ contains the interval with endpoints $2+i, 2-2 i$, and two half-lines included in $\{z: \operatorname{Re}(z)=1\}$. In order to define the endpoints of these lines and the curves joining these points to $2+i$ and $2-2 i$, we will use the above construction.

Let us apply the construction to the following disks:

$$
\begin{aligned}
& \bar{B}_{1}=\{z \in \mathbb{C}: \operatorname{Re}(z) \geq 2\} \cup\{\infty\}, \\
& \bar{B}_{2}=\bar{B}(3 / 2+i, 1 / 2), \\
& \bar{B}_{3}=\bar{B}(3 / 2+2 i, 1 / 2) .
\end{aligned}
$$

We get the sequence $\bar{B}_{n}$ for $n \geq 1$, the point $b \in\{z: \operatorname{Re}(z)=1\}$ and the curve $\gamma_{B}$ joining the tangency point of $\bar{B}_{1}$ and $\bar{B}_{2}$, namely $2+i$, to the point $b$ (note that $\left.\bar{B}_{4}=\bar{B}(9 / 8+3 i / 2,1 / 8)\right)$.

Let us take a second family of disks,

$$
\begin{aligned}
& \bar{D}_{1}=\bar{B}(3 / 2-i, 1 / 2), \\
& \bar{D}_{2}=\{z \in \mathbb{C}: \operatorname{Re}(z) \geq 2\} \cup\{\infty\}, \\
& \bar{D}_{3}=\bar{B}(3 / 2-2 i, 1 / 2) .
\end{aligned}
$$




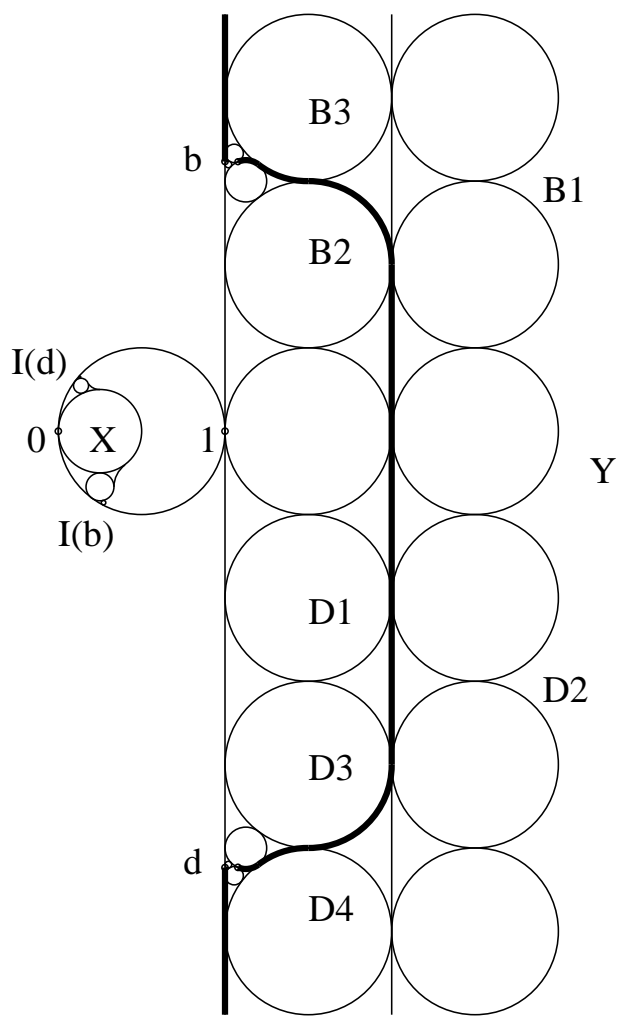

Fig. 3. The sets $X$ and $Y$

We obtain the sequence $\bar{D}_{n}$ for $n \geq 1$, the point $d \in\{z: \operatorname{Re}(z)=1\}$, and the curve $\gamma_{D}$ joining the point of tangency of $\bar{D}_{1}$ and $\bar{D}_{2}$, namely $2-i$, to the point $d$. In particular $\gamma_{D}$ contains the interval with endpoints $2-i$ and $2-2 i$ (note that $\bar{D}_{4}=\bar{B}(3 / 2-3 i, 1 / 2), \bar{D}_{5}=\bar{B}(9 / 8+5 i / 2,1 / 8)$ ).

The union of $\gamma_{B}, \gamma_{D}$ and the interval between $2+i$ and $2-i$ is a curve joining $b$ and $d$, which lies on the line $\{z: \operatorname{Re}(z)=1\}$. The rest of the boundary of $Y$ consists of the half-lines $\{z: \operatorname{Re}(z)=1 \wedge \operatorname{Im}(z) \geq b\}$, $\{z: \operatorname{Re}(z)=1 \wedge \operatorname{Im}(z) \leq d\}$ and the point at infinity.

For the set $X$, we take $\mathcal{I}(Y)$.

5. The properties of $\left\{\phi_{\sigma, \alpha}^{n, k}\right\}_{(n, k) \in I}$. In this section we prove that the family $\left\{\phi_{\sigma, \alpha}^{n, k}\right\}_{(n, k) \in I}$ defined by (1.4) and (1.5) for $\sigma \in \mathbb{R}, \alpha \in(0,1 / 2]$ forms an IFS and has the properties from Section 3. We also obtain some information about the limit set.

The main task is to verify OSC, and prove that the functions $\left\{\phi_{\sigma, \alpha}^{n, k}\right\}_{(n, k) \in I}$ defined on $X$ have values in $X$. To see this we will consider the maps which 
are conjugated to $\left\{\phi_{\sigma, \alpha}^{n, k}\right\}_{(n, k) \in I}$ by $\mathcal{I}$. By (4.1)-(4.3) we get

$$
\mathcal{I} \circ \phi_{\sigma, \alpha}^{n, k} \circ \mathcal{I}=\mathcal{I} \circ g_{\sigma, \alpha}^{n} \circ f^{k} \circ s \circ \mathcal{I}=T_{\sigma, \alpha}^{n, k} \circ \widetilde{\mathcal{I}}
$$

if $n \geq 1, k \in \mathbb{Z}$ or $n=0, k \leq-3$, and

$$
\mathcal{I} \circ \phi_{\sigma, \alpha}^{n, k} \circ \mathcal{I}=\mathcal{I} \circ g_{\sigma, \alpha}^{n} \circ f^{k} \circ s \circ f \circ \mathcal{I}=T_{\sigma, \alpha}^{n, k} \circ \tilde{\mathcal{I}} \circ T_{i}
$$

if $n=0, k \geq 2$, where $T_{i}$ denotes translation by $i$.

Because $X=\mathcal{I}(Y)$, in order to prove that the maps $\phi_{\sigma, \alpha}^{n, k}$ have values in $X$, it is enough to demonstrate that

$$
\left(T_{\sigma, \alpha}^{n, k} \circ \widetilde{\mathcal{I}}\right)(Y) \subset Y \quad\left(\text { or }\left(T_{\sigma, \alpha}^{n, k} \circ \widetilde{\mathcal{I}}\right)\left(T_{i}(Y)\right) \subset Y\right)
$$

In order to get OSC it is sufficient to prove that if $U=\operatorname{Int} Y$ then

$$
\left.\left(T_{\sigma, \alpha}^{n, k} \circ \widetilde{\mathcal{I}}\right)(U) \cap\left(T_{\sigma, \alpha}^{n^{\prime}, k^{\prime}} \circ \widetilde{\mathcal{I}}\right)(U)=\emptyset \quad \text { (or replace } U \text { by } T_{i}(U)\right),
$$

for distinct pairs $(n, k),\left(n^{\prime}, k^{\prime}\right) \in I$.

Let us see what the image of $Y=\mathcal{I}(X)$ under $\tilde{\mathcal{I}}$, and under $\widetilde{\mathcal{I}} \circ T_{i}$, looks like. Notice that the sets $\widetilde{\mathcal{I}}(Y)$ and $\left(\widetilde{\mathcal{I}} \circ T_{i}\right)(Y)$ are symmetrical, because the translation $T_{i}$ maps $Y$ onto a set symmetric with respect to the real axis.

The boundary of $\widetilde{\mathcal{I}}(Y)$ is a Jordan curve, containing the interval with endpoints $\widetilde{\mathcal{I}}(b), \widetilde{\mathcal{I}}(d)$, and the image of the interval between $2+i, 2-i$ which is an arc with endpoints $3 / 2-i / 2,3 / 2+i / 2$ (with 2 included). The remaining components are the images of $\gamma_{B}$ and $\gamma_{D}$ which will be considerd now.

The map $\widetilde{\mathcal{I}}$ preserves $\bar{H}$, and as it maps disks onto disks, we get the following

REMARK 5.1. The constructed sequence of disks which are tangent to $\partial \bar{H}$, and the separating curve, are invariant under $\widetilde{\mathcal{I}}$.

The images of $\bar{B}_{1}, \bar{B}_{2}$ and $\bar{B}_{3}$ (see (4.4)) are

$$
\begin{aligned}
& \widetilde{\mathcal{I}}\left(\bar{B}_{1}\right)=\bar{B}(3 / 2,1 / 2), \\
& \widetilde{\mathcal{I}}\left(\bar{B}_{2}\right)=\bar{B}(3 / 2-i, 1 / 2), \\
& \widetilde{\mathcal{I}}\left(\bar{B}_{3}\right)=\bar{B}(9 / 8-i / 2,1 / 8),
\end{aligned}
$$

whereas the images of $\bar{D}_{1}, \bar{D}_{2}, \bar{D}_{3}$ (see (4.5)) are

$$
\begin{aligned}
& \widetilde{\mathcal{I}}\left(\bar{D}_{1}\right)=\bar{B}(3 / 2+i, 1 / 2), \\
& \widetilde{\mathcal{I}}\left(\bar{D}_{2}\right)=\bar{B}(3 / 2,1 / 2), \\
& \widetilde{\mathcal{I}}\left(\bar{D}_{3}\right)=\bar{B}(9 / 8+i / 2,1 / 8) .
\end{aligned}
$$

Comparing $\widetilde{\mathcal{I}}\left(\bar{B}_{n}\right)$ with $\widetilde{\mathcal{I}}\left(\bar{D}_{n}\right)$, and $\widetilde{\mathcal{I}}\left(\bar{D}_{n}\right)$ with $\bar{D}_{n}$, using Remark 5.1 we get

REMARK 5.2. For every $n \geq 1$, the images of $\bar{B}_{n}$ and $\bar{D}_{n}$ differ by translation by $i$. It follows that the curves $\widetilde{\mathcal{I}}\left(\gamma_{B}\right)$ and $\tilde{\mathcal{I}}\left(\gamma_{D}\right)$ (joining $\widetilde{\mathcal{I}}(b)$ to 
$3 / 2-i / 2$ and $\widetilde{\mathcal{I}}(d)$ to $3 / 2+i / 2$ respectively) also differ by translation by $i$, in particular

$$
\widetilde{\mathcal{I}}(d)-\tilde{\mathcal{I}}(b)=i .
$$

REMARK 5.3. For every $n \geq 1$, the disk $\widetilde{\mathcal{I}}\left(\bar{D}_{n}\right)$ translated by $-3 i$ is equal to $\bar{D}_{n+2}$, and therefore the curve $\widetilde{\mathcal{I}}\left(\gamma_{D}\right)$ translated by $-3 i$ is included in $\gamma_{D}$, so we also get

$$
\widetilde{\mathcal{I}}(d)-3 i=d .
$$

Using Remark 5.2 we conclude that the curve $\widetilde{\mathcal{I}}\left(\gamma_{D}\right)$ is the intersection of the boundaries of the sets $\widetilde{\mathcal{I}}(Y)$ and $T_{i}(\widetilde{\mathcal{I}}(Y))$, which are Jordan domains. Because the remaining parts of the boundaries are disjoint, we conclude that upon translation by different multiples of $i$, the interiors of $\widetilde{\mathcal{I}}(Y)$ are disjoint. By symmetry, also the interiors of $\tilde{\mathcal{I}}\left(T_{i}(Y)\right)$ are disjoint after making different translations, which gives (5.4) in the case $n=n^{\prime}$.

To obtain (5.4) in the general case, note that because $Y$ is disjoint from $B(1,1)$ (see Figure 3 ), and is included in $\bar{H}$, we have $\widetilde{\mathcal{I}}(Y) \subset B(1,1) \cap \bar{H}$, so in particular

$$
\widetilde{\mathcal{I}}(Y) \subset\{z \in \mathbb{C}: 1 \leq \operatorname{Re}(z) \leq 2,-1 \leq \operatorname{Im}(z) \leq 1\} .
$$

So, if $n \neq n^{\prime}$ the images of $\operatorname{Int} \widetilde{\mathcal{I}}(Y)$ under $T_{\sigma, \alpha}^{n, k}=(1-\alpha) n / \alpha+i k+i \sigma n$ are disjoint provided that $(1-\alpha) / \alpha \geq 1$, which is satisfied for $\alpha \in(0,1 / 2]$. Thus, we get the open set condition for $\alpha \in(0,1 / 2]$.

Now we prove that the maps $\phi_{\sigma, \alpha}^{n, k}$ defined on $X$ have values in $X$ (condition (5.3)). For $n \geq 1,(5.3)$ is satisfied because $\left(T_{\sigma, \alpha}^{n, k} \circ \widetilde{\mathcal{I}}\right)(Y)$ is contained in the half-plane $\{z: \operatorname{Re}(z) \geq 1+n(1-\alpha) / \alpha\}$, hence for $\alpha \in(0,1 / 2]$ and $n \geq 1$ also in the half-plane $\{z: \operatorname{Re}(z) \geq 2\} \subset Y$. For $n=0$, it is enough to verify the cases of $k=-3$ and $k=2$.

For $n=0$ and $k=-3$ by Remark 5.3 the curve $T_{\sigma, \alpha}^{0,-3}\left(\widetilde{\mathcal{I}}\left(\gamma_{D}\right)\right)$ (translation by $-3 i$ ) is included in $\gamma_{D}$, hence in the boundary of $Y$. Because the remaining part of the boundary of $\widetilde{\mathcal{I}}(Y)$ after translation by $-3 i$ is also included in $Y$, we get

$$
\left(T_{\sigma, \alpha}^{0,-3} \circ \widetilde{\mathcal{I}}\right)(Y) \subset Y
$$

proving (5.3) for $n=0, k=-3$.

Let $n=0, k=2$. Because of the symmetry of the sets $Y, T_{i}(Y)$ and $\widetilde{\mathcal{I}}(Y), \widetilde{\mathcal{I}}\left(T_{i}(Y)\right)$ we get, by $(5.8)$,

$$
\left(T_{\sigma, \alpha}^{0,2+1} \circ \tilde{\mathcal{I}}\right)\left(T_{i}(Y)\right) \subset T_{i}(Y), \quad \text { so } \quad\left(T_{\sigma, \alpha}^{0,2} \circ \tilde{\mathcal{I}} \circ T_{i}\right)(Y) \subset Y,
$$

which gives (5.3) in this case. Note that $b$ is mapped under $T_{\sigma, \alpha}^{0,2} \circ \widetilde{\mathcal{I}} \circ T_{i}$ onto itself, so we get an equality similar to (5.6),

$$
\left(T_{\sigma, \alpha}^{0,2} \circ \tilde{\mathcal{I}} \circ T_{i}\right)(b)=\tilde{\mathcal{I}}(b+i)+2 i=b .
$$


Now we prove (3.1), which means that the maps $\phi_{\sigma, \alpha}^{n, k}$ are contractions with the same constant. If $\phi_{\sigma, \alpha}^{n, k}$ is defined by (1.4), it is enough to consider $F_{\sigma, \alpha}^{n, k}$ on the set $s(X)$ because $s$ is an isometry. By $(4.2), F_{\sigma, \alpha}^{n, k}$ is conjugated to $T_{\sigma, \alpha}^{n, k}$ by $\mathcal{I}$, and the modulus of the derivative of $\mathcal{I}$ depends only on the distance from zero. Thus, it is sufficient to see that the distance from zero of every point in $(\mathcal{I} \circ s)(X)$ increases under translation by $T_{\sigma, \alpha}^{n, k}$ more than $C$ times, for some $C \geq 1$. This is indeed true, because by $(4.3),(\mathcal{I} \circ s)(X)=$ $(\widetilde{\mathcal{I}} \circ \mathcal{I})(X)=\widetilde{\mathcal{I}}(Y)$, and it is enough to use $(5.7)$.

If $\phi_{\sigma, \alpha}^{n, k}$ is given by (1.5), then the previous argument does not work because of the composition with $f$. But in this case it is enough to consider the map $\phi_{\sigma, \alpha}^{0,2}$. We have

$$
\phi_{\sigma, \alpha}^{0,2}(z)=\frac{(i-1) z+1}{-(2+i) z+2 i+1} .
$$

One can verify that the modulus of the derivative is greater than or equal to one in the disk $\bar{B}\left(\frac{1+2 i}{2+i}, \frac{1}{\sqrt{5}}\right)$. Moreover, this disk is disjoint from the halfplane $\{z: \operatorname{Re}(z) \leq 1 / 2\}$ which contains $X$. Hence the maps $\phi_{\sigma, \alpha}^{n, k}$ are contractions with the same constant, and from the above considerations it follows that they are contractions on some neighborhood of $X$.

Now only conditions (1)-(4) from Section 3 remain to be verified. Condition (1) follows by construction, and the OSC condition for $U=\operatorname{Int}_{\mathbb{R}^{d}}(X)$ has already been checked. Condition (2) follows from the fact that the quotients of the diameters of the consecutive disks which were used to construct $\partial X$ are uniformly bounded. Condition (3) is satisfied because the maps $\phi_{\sigma, \alpha}^{n, k}$ are homographies. In order to prove (4) note that the derivatives of compositions of $\phi_{\sigma, \alpha}^{n, k}$ vanish only at the preimages of infinity. The maps $\phi_{\sigma, \alpha}^{n, k}$ are contractions on some neighborhood of $X$, so on that neighborhood the derivatives do not vanish, and hence using a version of the Koebe distortion theorem (see [3]), we obtain (4).

Corollary 5.4. The family of maps $\phi_{\sigma, \alpha}^{n, k}: X \rightarrow X$ forms a conformal IFS which satisfies the conditions from Section 3.

The closure of the limit set of the IFS will be denoted by $C_{\sigma, \alpha}$. Note that the closure differs from the limit set by a countable set of points which are preimages of infinity under compositions of $\phi_{\sigma, \alpha}^{n, k}$. It follows that the Hausdorff dimensions of the limit set and of its closure are equal.

Let

$$
l=\{z: \operatorname{Re}(z)=1 \wedge \operatorname{Im}(z) \geq b\} \cup\{z: \operatorname{Re}(z)=1 \wedge \operatorname{Im}(z) \leq d\} \cup\{\infty\} .
$$

Because $d$ and $b$ are fixed points of $T_{\sigma, \alpha}^{0,-3} \circ \widetilde{\mathcal{I}}$ and $T_{\sigma, \alpha}^{0,2} \circ \widetilde{\mathcal{I}} \circ T_{i}$ respectively, 
by (5.5) we get

$$
\bigcup_{k \leq-3}\left(T_{\sigma, \alpha}^{0, k} \circ \tilde{\mathcal{I}}\right)(l) \cup \bigcup_{k \geq 2}\left(T_{\sigma, \alpha}^{0, k} \circ \tilde{\mathcal{I}} \circ T_{i}\right)(l)=l \backslash\{\infty\} .
$$

After conjugation by $\mathcal{I}$, we have

$$
\overline{\bigcup_{(0, k) \in I} \phi_{\sigma, \alpha}^{0, k}(\mathcal{I}(l))}=\mathcal{I}(l) .
$$

Thus $l \in Y$, and so $\mathcal{I}(l) \subset X$. We get

Corollary 5.5. The set $\mathcal{I}(l)$ is contained in $C_{\sigma, \alpha}$.

Using (5.5) for fixed $n \geq 1$, we obtain

$$
\bigcup_{k \in \mathbb{Z}}\left(T_{\sigma, \alpha}^{n, k} \circ \tilde{\mathcal{I}}\right)(l)=\left\{z \in \mathbb{C}: \operatorname{Re}(z)=1+n \frac{1-\alpha}{\alpha}\right\},
$$

and so after applying $\mathcal{I}$, because $\mathcal{I}(l) \subset C_{\sigma, \alpha}$, we have

Corollary 5.6. The circle $S\left(\frac{\alpha / 2}{\alpha+n(1-\alpha)}, \frac{\alpha / 2}{\alpha+n(1-\alpha)}\right)$, which is the closure of $\mathcal{I}\left(\left\{z \in \mathbb{C}: \operatorname{Re}(z)=1+n \frac{1-\alpha}{\alpha}\right\}\right)$, is contained in $C_{\sigma, \alpha}$.

Taking $n=1$ we get the circle $S(\alpha / 2, \alpha / 2)$ of diameter $\alpha$. It is the largest circle contained in $C_{\sigma, \alpha}$. In further considerations the images of the disk $\bar{B}(\alpha / 2, \alpha / 2)$ under $\phi_{\sigma, \alpha}^{n, k}$ will be important. These images will be called the level-one disks, and denoted

$$
\bar{B}_{\sigma, \alpha}^{n, k}:=\phi_{\sigma, \alpha}^{n, k}(\bar{B}(\alpha / 2, \alpha / 2)) .
$$

Let us now consider the points $b$ and $d$. Set $b=1+i y_{b}$ and $d=1-i y_{d}$. Because $\widetilde{\mathcal{I}}$ acts on the line $\{z: \operatorname{Re} z=1\}$ as minus inversion, by (5.6) and (5.9) we get respectively

$$
-\frac{1}{-y_{d}}-3=-y_{d} \quad \text { and } \quad-\frac{1}{y_{b}+1}+3=y_{b} .
$$

Because $\left|y_{d}\right|>1$ and $\left|y_{b}\right|>1$, we conclude that

$$
y_{d}=\frac{3+\sqrt{5}}{2} \text { and } y_{b}=\frac{1+\sqrt{5}}{2} .
$$

The points $b$ and $d$ can also be described, in a natural way, via

$$
y_{d}=3-\frac{1}{3-\frac{1}{3-\cdots}}, \quad y_{b}=2-\frac{1}{3-\frac{1}{3-\cdots}} .
$$

6. The sum of $t$ th powers of quotients of diameters. In this section we obtain a formula for the diameters of level-one disks and consider the sum of the $t$ th powers of their quotients. 
For $\alpha \in(1 / 2,1)$, OSC obviously fails, so the setting does not have a clear geometric interpretation. Nevertheless the calculation below can be carried out for all $\alpha \in(0,1)$. Moreover, considering $\alpha$ close to one helps us draw a conclusion for $\alpha \in(0,1 / 2]$.

Set

$$
\beta:=\frac{\alpha}{1-\alpha} .
$$

This yields a one-to-one correspondence between $\alpha \in(0,1)$ and $\beta \in(0, \infty)$ $\left(\alpha=\frac{\beta}{\beta+1}\right)$, so we may use $\alpha$ and $\beta$ alternatively.

Now we will give a formula for the diameters of level-one disks. The disk $\bar{B}(\alpha / 2, \alpha / 2)$ is $f$-invariant, so we may consider both cases (1.4) and (1.5) together. The image of $\bar{B}(\alpha / 2, \alpha / 2)$ under $s$ is $\bar{B}(1-\alpha / 2, \alpha / 2)$, after inversion it is $\bar{B}(1+\beta / 2, \beta / 2)$; finally after making translations $T_{\sigma, \alpha}^{n, k}$ for $n, k \in I$, we obtain the family of disks

$$
\bar{B}\left(1+\frac{\beta}{2}+\frac{n}{\beta}+i(k+\sigma n), \frac{\beta}{2}\right)
$$

whose images under inversion are the level-one disks $\bar{B}_{\sigma, \alpha}^{n, k}$.

Notice that the diameter of $\bar{B}(z, r)$, where $|z|=R$, after inversion is

$$
\frac{1}{R-r}-\frac{1}{R+r}=\frac{2 r}{R^{2}-r^{2}},
$$

provided that $R>r$. For the disks $\bar{B}_{\sigma, \alpha}^{n, k}$ we have

$$
R^{2}=\left(1+\frac{\beta}{2}+\frac{n}{\beta}\right)^{2}+(k+\sigma n)^{2} \quad \text { and } \quad r=\frac{\beta}{2} .
$$

Hence

$$
\begin{aligned}
\operatorname{diam}\left(\bar{B}_{\sigma, \alpha}^{n, k}\right) & =\frac{\beta}{\left(1+\frac{n}{\beta}\right)^{2}+2\left(1+\frac{n}{\beta}\right) \frac{\beta}{2}+\frac{\beta^{2}}{4}+(k+\sigma n)^{2}-\frac{\beta^{2}}{4}} \\
& =\frac{\beta}{\left(1+\frac{n}{\beta}\right)^{2}+\beta\left(1+\frac{n}{\beta}\right)+(k+\sigma n)^{2}}
\end{aligned}
$$

The sum of the $t$ th powers of the quotients of the diameters of $\bar{B}_{\sigma, \alpha}^{n, k}$ and $B(\alpha / 2, \alpha / 2)$ will be denoted by

$$
Q_{\sigma, \alpha}(t):=\sum_{(n, k) \in I}\left(\frac{\operatorname{diam}\left(\bar{B}_{\sigma, \alpha}^{n, k}\right)}{\alpha}\right)^{t}=\sum_{(n, k) \in I}\left(\operatorname{diam}\left(\bar{B}_{\sigma, \alpha}^{n, k}\right) \frac{\beta+1}{\beta}\right)^{t} .
$$

Let

$$
P_{\sigma, \alpha}(t):=\log Q_{\sigma, \alpha}(t) .
$$

If the IFS consisted of affine maps, $P_{\sigma, \alpha}(t)$ would be the topological pressure. 
For fixed $\alpha \in(0,1)$ (as well $\beta$ ), there exist constants $C_{1}, C_{2}>0$ such that

$$
\frac{C_{1}}{\left(1+\frac{\beta}{2}+\frac{n}{\beta}\right)^{2}+(k+\sigma n)^{2}}<\frac{\operatorname{diam}\left(\bar{B}_{\sigma, \alpha}^{n, k}\right)}{\alpha}<\frac{C_{2}}{\left(1+\frac{\beta}{2}+\frac{n}{\beta}\right)^{2}+(k+\sigma n)^{2}} .
$$

Hence we can estimate the terms of the sum $Q_{\sigma, \alpha}(t)$, from above and below, by inverses, to the power $t$, of the distance from zero of points belonging to some lattice. We get the following

REMARK 6.1. $Q_{\sigma, \alpha}(t)$ is finite for $t>1$; furthermore, $Q_{\sigma, \alpha}(t)$ tends to infinity as $t \searrow 1$, which gives the strong regularity of IFS.

If we increase the exponent $t$ suitably, we could pass to the limit with $\widetilde{Q}_{\sigma, \alpha}(t)$ as $\beta \rightarrow \infty(\alpha \nearrow 1)$. Let us define a useful function $\widetilde{Q}_{\sigma, \alpha}$ as follows:

$$
\widetilde{Q}_{\sigma, \alpha}(t):=Q_{\sigma, \alpha}\left(\frac{1}{1-\alpha} t\right)=Q_{\sigma, \alpha}((\beta+1) t) .
$$

Proposition 6.2. For all $t>0$ and $\sigma \in \mathbb{R}$ the limit

$$
\lim _{\beta \rightarrow \infty} \widetilde{Q}_{\sigma, \alpha}(t)=: \widetilde{Q}_{\sigma}(t)
$$

exists, and

$$
\widetilde{Q}_{\sigma}(t)=\sum_{(n, k) \in I} e^{-t\left(n+(k+\sigma n)^{2}\right)} .
$$

Proof. We see from (6.1) that $\widetilde{Q}_{\sigma, \alpha}(t)$ is the sum of the following terms:

$$
\begin{gathered}
\left(\frac{\operatorname{diam}\left(\bar{B}_{\sigma, \alpha}^{n, k}\right)}{\alpha}\right)^{(\beta+1) t}=\left(\frac{\beta+1}{\left(1+\frac{n}{\beta}\right)^{2}+\beta\left(1+\frac{n}{\beta}\right)+(k+\sigma n)^{2}}\right)^{(\beta+1) t} \\
=\left(1+\frac{n+(k+\sigma n)^{2}}{\beta+1}+\frac{2 n}{\beta(\beta+1)}+\frac{n^{2}}{\beta^{2}(\beta+1)}\right)^{-(\beta+1) t}
\end{gathered}
$$

Thus

$$
\lim _{\beta \rightarrow \infty}\left(\frac{\operatorname{diam}\left(\bar{B}_{\sigma, \alpha}^{n, k}\right)}{\alpha}\right)^{(\beta+1) t}=e^{-t\left(n+(k+\sigma n)^{2}\right)},
$$

so the terms of $Q_{\sigma, \alpha}(t)$ tend to respective terms of (6.2). Hence, in order to prove the convergence of $\widetilde{Q}_{\sigma, \alpha}(t)$ as $\beta \rightarrow \infty$, it is sufficient to show that for all $t>0$,

$$
\lim _{\beta \rightarrow \infty} \sum_{(n, k) \in I}\left(\frac{\operatorname{diam}\left(\bar{B}_{\sigma, \alpha}^{n, k}\right)}{\alpha}\right)^{(\beta+1) t}=\sum_{(n, k) \in I} \lim _{\beta \rightarrow \infty}\left(\frac{\operatorname{diam}\left(\bar{B}_{\sigma, \alpha}^{n, k}\right)}{\alpha}\right)^{(\beta+1) t} .
$$

Fix $\beta_{0}$. Using (6.3), for $\beta>\beta_{0}$ each term of the sum $Q_{\sigma, \alpha}(t)$ can be estimated 
from above by

$$
\left(1+\frac{n+(k+\sigma n)^{2}}{\beta+1}\right)^{-(\beta+1) t}<\left(1+\frac{n+(k+\sigma n)^{2}}{\beta_{0}+1}\right)^{-\left(\beta_{0}+1\right) t}
$$

Because of convergence of terms, to get (6.4), it is enough to prove that for all $t>0$ there exists $\beta_{0}$ such that

$$
\sum_{(n, k) \in I}\left(1+\frac{n+(k+\sigma n)^{2}}{\beta_{0}+1}\right)^{-\left(\beta_{0}+1\right) t}<\infty
$$

For fixed $\beta_{0}$ and $(n, k) \in I$, we have

$$
\begin{aligned}
& \left(1+\frac{n+(k+\sigma n)^{2}}{\beta_{0}+1}\right)^{-\left(\beta_{0}+1\right) t}=\left(\frac{\beta_{0}+1}{\beta_{0}+1+n+(k+\sigma n)^{2}}\right)^{\left(\beta_{0}+1\right) t} \\
& <\left(\frac{\beta_{0}+1}{n+(k+\sigma n)}\right)^{\left(\beta_{0}+1\right) t}<\left(\beta_{0}+1\right)^{\left(\beta_{0}+1\right) t}\left(\frac{1}{\sqrt{n^{2}+(k+\sigma n)^{2}}}\right)^{\left(\beta_{0}+1\right) t} .
\end{aligned}
$$

Choosing $\beta_{0}$ so that $\left(\beta_{0}+1\right) t>2$ yields $(6.5)$.

7. *Property. If $f \in L^{1}(\mathbb{R})$ and $\operatorname{Var}(f)<\infty$, then we can define a periodic function $S_{f}: \mathbb{R} \rightarrow \mathbb{R}$ as follows:

$$
S_{f}(\sigma)=\sum_{k \in \mathbb{Z}} f(k+\sigma) .
$$

We say that $f$ has the ${ }^{*}$ property if the function $S_{f}$ is constant. In that case we have $S_{f}(\sigma)=\int_{\mathbb{R}} f(x) d x$.

Lemma 7.1. If $f, g \in L^{1}(\mathbb{R})$ and $\operatorname{Var}(f), \operatorname{Var}(g)<\infty$, then

$$
S_{f \star g}=f \star S_{g}=g \star S_{f},
$$

and it follows that if $f$ or $g$ has the ${ }^{*}$ property, then so does $f \star g$.

Proof. Let us prove the first equality:

$$
\begin{aligned}
S_{f \star g}(\sigma) & =\sum_{k \in \mathbb{Z}}(f \star g)(k+\sigma)=\sum_{k \in \mathbb{Z}} \int_{\mathbb{R}} f(t) g(k+\sigma-t) d t \\
& =\int_{\mathbb{R}} f(t) \sum_{k \in \mathbb{Z}} g(k+\sigma-t) d t=\int_{\mathbb{R}} f(t) S_{g}(\sigma-t) d t=\left(f \star S_{g}\right)(\sigma) .
\end{aligned}
$$

The second equality comes from the commutativity of convolution:

$$
f \star S_{g}=S_{f \star g}=S_{g \star f}=g \star S_{f} .
$$

The last assertion follows from the fact that convolution with a constant function is constant. 
For $m, \varrho \in \mathbb{R}, \varrho \neq 0$, let $g_{m, \varrho}$ denote the normal probability distribution function,

$$
g_{m, \varrho}=\frac{1}{\sqrt{2 \pi} \varrho} e^{-(x-m)^{2} / 2 \varrho^{2}} .
$$

REMARK 7.2. It is well known that convolution of two such distributions is still a normal probability distribution,

$$
g_{m, \varrho} \star g_{\widetilde{m}, \widetilde{\varrho}}=g_{m+\widetilde{m}, \varrho+\widetilde{\varrho}} .
$$

LEMma 7.3. No distribution $g_{m, \varrho}$ has the ${ }^{*}$ property.

Proof. We may assume that $m=0$, and $g_{0, \varrho}$ will be denoted by $g_{\varrho}$. Notice that for $\varrho \leq 1 / \sqrt{2 \pi}$ we have

$$
S_{g_{\varrho}}(0)>g_{\varrho}(0)=\frac{1}{\sqrt{2 \pi} \varrho} \geq 1=\int_{\mathbb{R}} g_{\varrho} d x,
$$

so that $g_{\varrho}$ does not have the ${ }^{*}$ property, because $S_{g_{\varrho}}(0) \neq \int_{\mathbb{R}} g_{\varrho} d x$.

For $\varrho>1 / \sqrt{2 \pi}$ we argue by contradiction. Assume that $g_{\widetilde{\varrho}}$ has the * property for some $\widetilde{\varrho}$. Considering convolution of $g_{\widetilde{\varrho}}$ and $g_{\varrho}$ for $\varrho>0$, and using Remark 7.2 and Lemma 7.1, we get the * property for $g_{\varrho}$ for all $\varrho \geq \widetilde{\varrho}$.

We may treat $S_{g_{\varrho}}(\sigma)$ as a real-analytic function of $\varrho$ and $\sigma$. The * property of $g_{\varrho}$ for $\varrho \geq \widetilde{\varrho}$ means that for $\varrho \geq \widetilde{\varrho}$ the values of $S_{g_{\varrho}}(\sigma)$ do not depend on $\sigma$. But, thanks to analyticity, for $\widetilde{\varrho}>\varrho>0$ the values of $S_{g_{\varrho}}(\sigma)$ also do not depend on $\sigma$, which gives the ${ }^{*}$ property for $\varrho \leq 1 / \sqrt{2 \pi}$, a contradiction.

REMARK 7.4. One can give a different proof using Fourier series and Fourier transform.

\section{The dependence on the parameter}

COROLlaRY 8.1. For any fixed $t$, the limit function $\widetilde{Q}_{\sigma}(t)$ is not constant with respect to $\sigma$.

Proof. Let $G_{t}(x)=e^{-t x^{2}}$. Then

$$
\widetilde{Q}_{\sigma}(t)=\sum_{(n, k) \in I} e^{-t\left(n+(k+\sigma n)^{2}\right)}=\sum_{(0, k) \in I} e^{-t k^{2}}+\sum_{n \geq 1} e^{-t n} S_{G_{t}}(\sigma n) .
$$

The first sum does not depend on $\sigma$, so we may only consider the second.

The function $S_{G_{t}}(\sigma)$ is even (because $G_{t}$ is even) and by Lemma 7.3 we know that it is not constant. Because $S_{G_{t}}(\sigma)$ is analytic, we conclude that it attains an extremum at zero. Let $s$ (even) be the order of the first non-vanishing derivative at zero. The functions $S_{G_{t}}(\sigma n)$ for $n \geq 1$ attain the same extremum at zero as $S_{G_{t}}(\sigma)$, so the derivatives of order $s$ are also the first non-vanishing ones, and have the same sign. 
We may differentiate the sum

$$
\sum_{n \geq 1} e^{-t n} S_{G_{t}}(\sigma n)
$$

term by term, so the derivatives up to order $s-1$ all vanish at zero. Because the coefficients $e^{-t n}$ are positive, the derivative of order $s$ is not zero, which means that (8.1) attains an extremum at $\sigma=0$, and so $\widetilde{Q}_{\sigma}(t)$ is not constant.

THEOREM 8.2. For every $c>0$, the equation $Q_{\sigma, \alpha}(t)=c\left(P_{\sigma, \alpha}(t)=\right.$ $\log c$ ) defines uniquely a function $t_{c}(\sigma, \alpha)$. For fixed $c>0$, the set of parameters $\alpha$ for which the values of $t_{c}(\sigma, \alpha)$ do not depend on $\sigma$ (which may be empty) has no accumulation points in $(0,1]$.

Proof. The function $\widetilde{Q}_{\sigma, \alpha}(t)=Q_{\sigma, \alpha}\left(\frac{1}{1-\alpha} t\right)=Q_{\sigma, \alpha}((\beta+1) t)$ may be considered as an analytic function of three variables $\sigma, \alpha, t$ defined on the set $\sigma \in \mathbb{R},(\beta+1) t>1, t>0$. Moreover $\widetilde{Q}_{\sigma, \alpha}$ extends as a continuous function to the set $\alpha=1(\beta=\infty), t>0, \sigma \in \mathbb{R}$.

If we fix $\sigma \in \mathbb{R}$ and $\alpha \in(0,1]$, then $\widetilde{Q}_{\sigma, \alpha}$ is a strictly decreasing function of $t$ and attains all values greater than zero. Hence by the implicit function theorem, the equation $\widetilde{Q}_{\sigma, \alpha}=c$ defines a function $\widetilde{t}_{c}(\alpha, \sigma)$ for $\alpha \in(0,1)$, $\sigma \in \mathbb{R}$. Moreover, $\widetilde{t}_{c}(\alpha, \sigma)$ extends to the set $\alpha=1$.

Because $\widetilde{Q}_{\sigma}$ is not constant for any $t$, we can find two parameters $\sigma_{1}, \sigma_{2}$ so that $\widetilde{t}_{c}\left(\alpha, \sigma_{1}\right)$ and $\widetilde{t}_{c}\left(\alpha, \sigma_{2}\right)$ are distinct analytic functions of $\alpha$. Then the set of solutions of $\widetilde{t}_{c}\left(\alpha, \sigma_{1}\right)-\widetilde{t}_{c}\left(\alpha, \sigma_{2}\right)=0$ cannot have accumulation points, so the value of $\widetilde{t}_{c}(\alpha, \sigma)$ may be independent of $\sigma$ at most for parameters from a set of $\alpha \in(0,1]$ without accumulation points.

The function $t_{c}(\alpha, \sigma)=\widetilde{t}_{c}(\alpha, \sigma) /(\beta+1)$ is a solution of $Q_{\sigma, \alpha}(t)=c$, thus the proof is finished.

REMARK 8.3. The homographies $\phi_{\sigma, \alpha}^{n, k}$ could be replaced by affine conformal functions, mapping $\bar{B}(\alpha / 2, \alpha / 2)$ onto the level-one disks. Unfortunately, it is not clear whether (and for which parameters $\alpha$ ) the open set condition (OSC) is satisfied, and whether the fundamental domain is mapped into itself. The latter in fact can be ensured by increasing the fundamental domain. If we knew it, we would draw the conclusion that the Hausdorff dimension of the limit set does not depend on $\sigma$ at most for parameters $\alpha$ from a set without accumulation points.

Indeed, for such an affine IFS the function $P_{\sigma, \alpha}(t)=\log Q_{\sigma, \alpha}(t)$ would be the topological pressure. Therefore the Hausdorff dimension would be the solution of the equation $Q_{\sigma, \alpha}(t)=1$. By Theorem 8.2 the function $t_{1}(\sigma, \alpha)$ which is the solution of this equation does not depend on $\sigma$ at most for $\alpha$ 's from a set without accumulation points. 


\section{References}

[1] A. Douady, Does a Julia set depend continuously on the polynomial?, in: Proc. Sympos. Appl. Math. 49, Amer. Math. Soc., 1994, 91-135.

[2] A. Douady, P. Sentenac et M. Zinsmeister, Implosion parabolique et dimension de Hausdorff, C. R. Acad. Sci. Paris Sér. I 325 (1997), 765-772.

[3] E. Hille, Analytic Function Theory, Ginn and Co., Boston, 1962.

[4] R. D. Mauldin and M. Urbański, Dimensions and measures for infinite iterated function systems, Proc. London Math. Soc. 3 (1996), 105-154.

[5] D. Ruelle, Repellers for real analytic maps, Ergodic Theory Dynam. Systems 2 (1982), 99-107.

[6] M. Urbański et M. Zinsmeister, Continuity of Hausdorff dimension of Julia-Lavaurs sets as a function of the phase, Conform. Geom. Dyn. 5 (2001), 140-152.

Ludwik Jaksztas

Institute of Mathematics

Polish Academy of Sciences

Śniadeckich 8

00-956 Warszawa, Poland

E-mail: jaksztas@impan.gov.pl
Faculty of Mathematics and Information Sciences Warsaw University of Technology Pl. Politechniki 1 00-661 Warszawa, Poland

Received March 21, 2006;

received in final form March 27, 2007 